\title{
PROBLEMS AND PITFALLS
}

\section{Diagnosis and management of common maxillofacial injuries in the emergency department. Part 5: dentoalveolar injuries}

\author{
P Ó Ceallaigh, K Ekanaykaee, C J Beirne, D W Patton
}

Emerg Med J 2006;24:429-430. doi: 10.1136/emj.2006.035949

Injuries to teeth can be very distressing for patients. Prompt treatment is essential. Injuries of the tooth bearing portion of the mandible are common and can even result after a relatively low impact trauma. The alveolus (tooth bearing portion of bone) and/or the tooth may be damaged. Segmental fractures involve multiple teeth and the supporting alveolar bone.

\section{INITIAL MANAGEMENT}

Treatment of alveolar bone fractures requires manually repositioning the segment of displaced teeth back into proper alignment. The patient should be promptly referred to the maxillofacial service. This may be done under local anaesthetic. Dental impressions are taken and a splint is fabricated and applied.

\section{Avulsed teeth}

Deciduous teeth-that is baby teeth-should not be reimplanted as to do so may cause further injury to the developing permanent teeth. The average ages of eruption of permanent dentition in years is shown in fig 2. Reimplantation of the avulsed teeth is unnecessary in children less than six years of age.

Reimplantation of permanent tooth should be carried immediately, as periodontal ligament cells, which attach the tooth to the bone, necrose after 2 hours. The goal of reimplantation is to preserve the periodontal ligament.

Contact with the root of the tooth is best avoided to prevent further damage to the periodontal ligament.

Hold the tooth by the crown and gently wash it in saline. Do not attempt to sterilise the tooth. Do not remove the clot formed in the socket prior to reimplantation of the avulsed tooth. Replace the tooth in the socket. Compress the buccal and palatal/lingual alveolar plates. If the tooth does not seat easily, get the patient to bite on some gauze. The patient should be commenced on oral antibiotics (metronidazole), analgesia, and chlorohexidine mouth wash. Tetanus prophylaxis should be administered if the patient's immunisation is not up to date.

The tooth should not be allowed to dry under any circumstances as the periodontal ligament will die. Ideally the tooth should be reimplanted within five minutes but if this is not possible it should be stored in a medium that will help maintain vitality of the periodontal ligament fibres.

\section{Storage media}

Saliva is always available (place tooth in buccal sulcus of the patient's mouth).

Beware of doing so in a child as there is a risk of aspiration of the tooth. Ice cooled milk is the next best temporary storage medium of the avulsed teeth. Saline should be used as a last resort.

All intraoral lacerations must be cleaned and explored, looking for any foreign bodies. The oral 


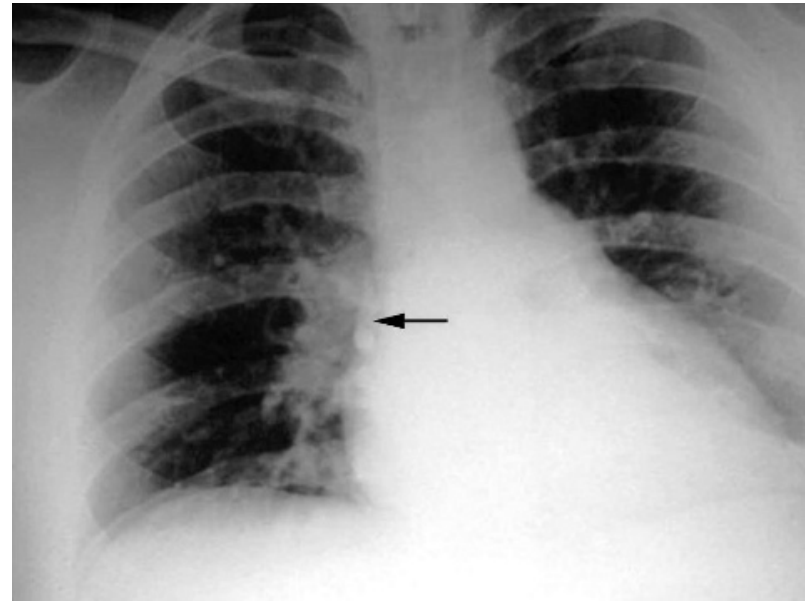

Figure 1 Chest radiograph showing lodged tooth in the right main bronchus

frenum, if torn, will heal without long term consequences.

Lacerations of the lips may be sutured. Local anaesthetic ( $2 \%$ lignocaine with 1: 80000 adrenaline) is administered. The lips must be thoroughly inspected and washed to ensure there is no buried foreign material-for example, tooth. Vicryl is the suture of choice.

\section{ACKNOWLEDGEMENTS}

The authors would like to thank Simon Edwards, Medical Illustrator, Morriston Hospital, and Michelle and Ronan for their cooperation in the production of this article.

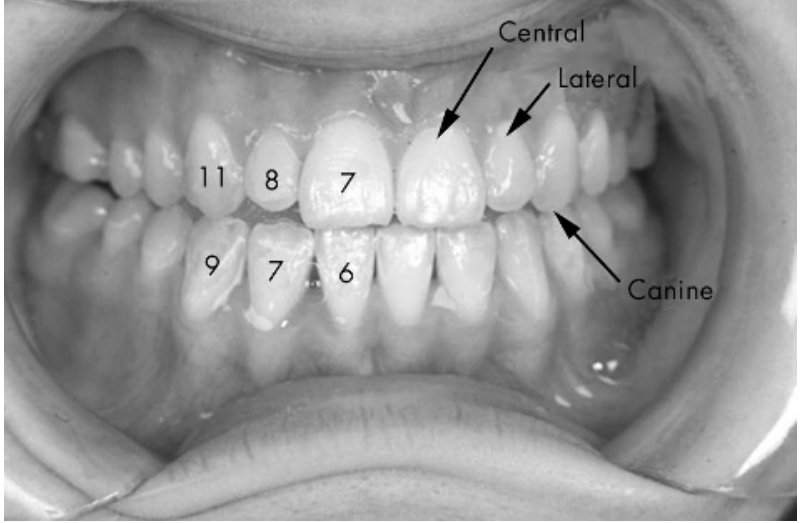

Figure 2 Average ages of eruption of permanent dentition in years

\section{Suggested further reading}

Andreasen JO, Andreasen FM. Essentials of traumatic injuries to the teeth. 2nd edition. Copenhagen, Denmark: Munksgaard and Mosby, 2000.

\section{Authors' affiliations}

K Ekanaykaee, C J Beirne, Beaumont Hospital, Dublin, Eire P Ó Ceallaigh, D W Patton, Morriston hospital, Swansea, Wales, UK Competing interests: None.

\section{Stay a step ahead with Online First}

We publish all our original articles online before they appear in a print issue. This means that the latest clinical research papers go straight from acceptance to your browser, keeping you at the cutting edge of medicine. We update the site weekly so that it remains as topical as possible. Follow the Online First link on the home page and read the latest research. 